\title{
3 Evaluating the argument
}

Are our two additional assumptions-the meta-axiological assumption that the relevant (m-)value facts satisfy our axioms, and the conceptual assumption constituted by the prospect-explications-plausible? That is, do our axiologies and the m-value facts satisfy the conditions of the Expected Value Theorem? And are the prospect-explications viable definitions of cardinal value comparisons? In this chapter, I'll begin to answer these questions.

A conclusive answer, especially to the first question, is beyond the scope of this book. Each of the theorem's conditions is disputed, at least in standard decision theory and social choice theory. I can't defend any of them conclusively. So what I'll mostly do is compare their plausibility in the context of axiological uncertainty with their plausibility in other contexts-specifically, that of decision theory or first-order axiology, and (in the case of the Pareto Condition) that of social choice theory. I'll start with the Pareto Condition (Section 3.1) and the von Neumann-Morgenstern axioms (Section 3.2), and then discuss the prospectexplications (Section 3.3). I'll end the chapter by highlighting two interesting implications of this discussion for decision theory as a general theory of preferences, and for EVM as a higher-order theory of uncertainty about theories of axiological uncertainty (Section 3.4).

\subsection{The Pareto Condition}

Recall what the Pareto Condition says: for any two prospects with the same underlying probability distribution over axiologies, if they're equally good on all theories with nonzero probability, they're equally m-good, and if one of them is at least as good as the other on all theories with nonzero probability and strictly better on some, it's strictly m-better. Intuitively, this sounds very plausible. However, it's an ex ante Pareto requirement. It's concerned not with the betterness of outcomes, but with the betterness of prospects. For this reason especially, one may have doubts about it. Pareto conditions are rarely discussed in decision theory. But as mentioned (with respect to Harsanyi), there's an analogy between the theory of axiological uncertainty and social choice theory. We're concerned with an overall $\mathrm{m}$-value ordering that depends on the value-orderings of axiologies; social choice theory is concerned with a 'general betterness' ordering that depends on the 'individual betterness' orderings of people. ${ }^{56}$ And it's controversial whether,

56 Or at any rate, that is one interpretation of it. See particularly Broome (1991).

Ә Open Access. (c) 2021 Stefan Riedener, published by De Gruyter. (cc))BY-NC-ND This work is licensed under the Creative Commons Attribution-NonCommercial-NoDerivatives 4.0 International License.

https://doi.org/10.1515/9783110736199-003 
if one prospect is ex ante at least as good for all individuals as another and strictly better for some, it's ceteris paribus generally better. So we must be careful to assume such a condition in our context.

The most important argument against the ex ante Pareto condition for individual goodness is due to Marc Fleurbaey and Alex Voorhoeve (2013). It shows that this condition is in tension with (ex post) egalitarianism, the view that it's good if people fare equally well. Suppose we can either let both Antonio and Leonore end up with 20 units of good (prospect $f$ ), or conduct a lottery yielding, with equal probability, 31 units of good for Antonio and 10 such units for Leonore or vice versa (prospect $g$ ):

Tab. 3.1: Example to illustrate ex ante Pareto condition for individual goodness.

\begin{tabular}{rrrrrr}
\hline & \multicolumn{2}{c}{$\boldsymbol{f}$} & & \multicolumn{2}{c}{$\boldsymbol{g}$} \\
\cline { 2 - 3 } \cline { 5 - 6 } & Antonio & Leonore & & Antonio & Leonore \\
\hline 0.5 & 20 & 20 & 10 & 31 \\
0.5 & 20 & 20 & & 31 & 10 \\
\hline
\end{tabular}

Suppose that individual goodness is expectational. Then $g$ is ex ante better than $f$ for both Antonio and Leonore. So if general goodness satisfies an ex ante Pareto condition with respect to personal goodness, $g$ is ex ante better than $f$. However, according to (a relevant form of) egalitarianism, a state in which both Antonio and Leonore have 20 units of good is better than a state in which one has 31 and the other 10. So if it was certain that $g$ would lead to 31 for Antonio and 10 for Leonore, $f$ would ex ante be better. And if it was certain that $g$ would lead to 10 for Antonio and 31 for Leonore, $f$ would also be better. In other words, whatever the outcome of $g$ will be, if it was certain, $f$ would ex ante be better. Therefore, Fleurbaey and Voorhoeve claim, $f$ is ex ante better even if $g$ 's outcome is uncertain. This is because of the 'Principle of Full Information' (or basically Savage's 'Sure-Thing Principle,57): 'When one knows that, in every state of the world with positive probability, one would rightly rank two alternatives in a particular way, then one should so rank them' $(2013,120)$.

This may be a good reason to reject the ex ante Pareto condition concerning individual goodness. I won't explore this. What matters for our purposes is that there doesn't seem to be a plausible parallel reasoning about m-value. Fleurbaey and Voorhoeve's argument depends on the egalitarian claim that $f$ would

57 See Savage (1954, $21 \mathrm{ff}$.$) .$ 
be better than $g$ if $g$ 's outcome was certain. But it's not clear why an analogous premise should hold in the context of axiological uncertainty. Consider the following choice between $\boldsymbol{f}$ and $\boldsymbol{g}$ :

Tab. 3.2: Example to illustrate Pareto Condition.

\begin{tabular}{rrrrr}
\hline \multicolumn{2}{c}{$f$} & & \multicolumn{2}{c}{$g$} \\
\cline { 5 - 6 } $\boldsymbol{T}_{\mathbf{1}}$ & $\boldsymbol{T}_{\mathbf{2}}$ & & $\boldsymbol{T}_{1}$ & $\boldsymbol{T}_{2}$ \\
$\boldsymbol{p}_{\mathbf{1}} \mathbf{0 . 5}$ & $\boldsymbol{p}_{\mathbf{2}}=\mathbf{0 . 5}$ & & $\boldsymbol{p}_{1}=\mathbf{0 . 5}$ & $\boldsymbol{p}_{\mathbf{2}}=\mathbf{0 . 5}$ \\
\hline 20 & 20 & & 31 & 10 \\
\hline
\end{tabular}

Why should $\boldsymbol{f}$ be $\mathrm{m}$-better? What immediately comes to mind is risk-aversion-the view I expressed as

$$
\boldsymbol{a} \geq_{m} \boldsymbol{b} \quad \text { iff } \quad \sum_{i \in I} p_{i}^{\boldsymbol{a}} \rho\left(V_{i}^{\boldsymbol{a}}\right) \geq \sum_{i \in I} p_{i}^{\boldsymbol{b}} \rho\left(V_{i}^{\boldsymbol{b}}\right) .
$$

For some suitable increasing, strictly concave function $\rho$ (e.g., $\rho(x)=\sqrt{x}$ ), this view indeed implies that $\boldsymbol{f} \succeq_{m} \boldsymbol{g}$. However, we can't use it to argue against the Pareto Condition. As is easily verified, (3.1) actually satisfies this condition.

To argue against the Pareto Condition, we'd have to endorse a different form of risk-aversion. Say that $\mathrm{m}$-value is ex post risk-averse if there's an increasing strictly concave function $\rho$, such that

$$
\boldsymbol{a} \geq_{m} \boldsymbol{b} \quad \text { iff } \quad \sum_{i \in I, x \in X} \boldsymbol{a}(i, x) \rho\left(G_{i}(x)\right) \geq \sum_{i \in I, x \in X} \boldsymbol{b}(i, x) \rho\left(G_{i}(x)\right) .
$$

In (3.2), $\rho$ is applied not to the prospects that our options represent on given axiologies, but to outcomes. If m-value is ex post risk-averse, it doesn't violate Independence, as the theory defined in (3.1) does. On the other hand, as is again easily verified, it does violate the Pareto Condition, and imply that $\boldsymbol{f} \geq_{m} \mathbf{g}$ (for some $\rho$ ). So (3.2) might be used in an argument against the Pareto Condition. However, it's a very dubious view. Note what it implies if you're certain of one theory. Suppose you're certain of $T_{1}$, and face $\boldsymbol{k}$ and $\boldsymbol{l}: \boldsymbol{k}$ leads to an outcome of value 20 (according to $T_{1}$ ), and $\boldsymbol{l}$ with equal probability either to an outcome of value 10 , or to one of value 31 (according to $T_{1}$ ). Since you're certain of $T_{1}$, if goodness is expectational at the level of axiologies, you're certain that $\boldsymbol{l}$ is better than $\boldsymbol{k}$. Yet, (for the relevant $\rho$ ) (3.2) implies that $\boldsymbol{k}$ is $\mathrm{m}$-better. So it says that a prospect can be $\mathrm{m}$-better than another, even if it's certainly worse. This is very implausible. Surely, if we're certain that one axiology is true, we ought to rank prospects in accordance with it. 
Perhaps there are other reasons, apart from these forms of risk-aversion, for believing that $\boldsymbol{f}$ is $\mathrm{m}$-better than $\boldsymbol{g}$, and for denying the Pareto Condition on that basis. But I can't think of any plausible candidate. So I tentatively conclude there's no analogue to Fleurbaey and Voorhoeve's objection in the context of axiological uncertainty. As far as their objection is concerned, the condition seems plausible for m-value. Similarly, perhaps there are other kinds of objections to our Pareto Condition, other than reasons that parallel the egalitarian objection. But I can't see any I find remotely convincing. ${ }^{58}$ So as far as I see, in our context the ex ante Pareto Condition is indeed very plausible. It's a major drawback for a theory to violate it.

\section{2 vNM-conformability}

\section{The von Neumann-Morgenstern axioms as conditions on value}

Let's turn to the other part of our (meta-)axiological assumption, the von Neumann-Morgenstern axioms. There are two levels at which these appear in our theorem: as conditions on our axiologies, and as conditions on m-value. Consider first the assumption that all axiologies are vNM-conformable. Is this plausible? As a claim about all possible axiologies, or even all axiologies we should have some nonzero credence in, it's clearly false. There are non-vNM-conformable axiologies, and in some of them we should have at least some nonzero credence. However, this doesn't undermine the relevance of our theorem altogether. We should

58 There's a second standard objection against ex ante Pareto conditions in social choice theory. Some authors find ex ante Pareto improvements problematic when they depend on differences in people's beliefs (see e. g. Gilboa et al. 2014 or Mongin and D’Aspremont 1998, 442). Suppose Leonore and Antonio have an apple and an orange each, but Leonore doesn't like apples at all, and Antonio doesn't like oranges at all. In this case, there's a Pareto superior state in which Leonore gets both oranges and Antonio gets both apples. This state is possible because the two have different tastes, and it seems clearly preferable from the point of view of general goodness. But now suppose Felix is certain that it will rain tomorrow, and Mariane is certain that it will snow, and they both own $€ 100$ (which they cherish). Relative to their beliefs, it's ex ante better for both of them to agree to the bet in which Felix will receive Mariane's $£ 100$ if it rains, and Mariane will receive Felix’s £100 if it snows. However, since one of them clearly has false beliefs, there's something problematic about that agreement. That's especially true, say, if one of them was intentionally deceived. However, this objection doesn't carry over to our context either. I do allow that the probability distribution over outcomes in a prospect in $\mathcal{Q}$ may differ from theory to theory. But that's not because axiologies themselves somehow assign different probabilities to outcomes. I'm simply stipulating these probabilities. So whatever exactly we find problematic about belief-relative Pareto improvements, this won’t apply to our condition. 
simply see this condition as restricting its scope. Our result isn't a fully general theorem of axiological uncertainty. It's a theorem concerning uncertainty about vNM-conformable axiologies only. That's why I haven't stated this as part of the main conditional of the theorem, but as an antecedent supposition ('Suppose that all our $\geq_{i}$ are vNM-conformable'). It's not surprising that our theorem isn't fully general. The biconditional (2.10) is a theory of both axiological and descriptive uncertainty. It says you should maximise expected value across axiologies and descriptive states of nature, in a vNM-conformable manner. But that's implausible if one of our axiologies isn't vNM-conformable. If you were certain of such an axiology, you should arguably judge a prospect m-better than another if and only if it's better according to that axiology. So if that axiology isn't vNM-conformable, the m-value relation isn't vNM-conformable either. ${ }^{59}$ As a general theory of both axiological and descriptive uncertainty, a vNM-conformable theory of m-value is plausible at best for vNM-conformable axiologies.

How significant a restriction is this, or how common and plausible are nonvNM-conformable axiologies? For three of our four axioms, the relevant restrictions don't seem all too serious. It's been disputed that the betterness relation even satisfies Transitivity. ${ }^{60}$ I won't enter this debate here. But I think it's an analytic fact that 'better than' and 'at least as good as' are transitive. 'Better' is the comparative of 'good', and all comparatives are transitive. ${ }^{61}$ So I've been assuming as a matter of definition that an axiology is a transitive binary relation.

I take it that there are axiologies that strictly violate Independence. For instance, some views are risk-averse in a way that's strictly inconsistent with this axiom. And plausibly, we should have some nonzero credence in some such axiologies. ${ }^{62}$ So the assumption that all axiologies satisfy this axiom is a nontrivial restriction of our framework. Also, it's an essential aspect of it. There are alternative frameworks allowing for other risk-attitudes, potentially grounding theories other than Expected Value Maximisation, and certainly well worth exploring. ${ }^{63}$ But Independence is key for the framework and argument in this book.

59 In fact, given a suitable Pareto condition, $\geq_{m}$ then can't be vNM-conformable. The Pareto Condition on page 26 is slightly too weak to guarantee this. It doesn't rule out that the m-value relation may represent a complete sharpening of an underlying axiology that is incomplete. But a slightly stronger condition (like the one on page 115) would do.

60 See most prominently Temkin (2012); also Rachels (1998).

61 This view is defended in Broome (2004, ch.4); see e. g. Binmore and Voorhoeve (2003) or Voorhoeve (2013) for further defences of the transitivity of betterness.

62 See e. g. Buchak (2013) for a defence of risk-sensitivity in decision theory.

63 See most prominently Buchak (2013); also Dietrich and Jabarian $(2018$; 2021) for the theory of normative uncertainty. 
However, my sense is that axiologies that strictly violate Independence are comparatively implausible. That's for two reasons. First, as indicated in Section 2.4 (on page 36), Independence seems to express a compelling thought. If one prospect is better than another, then lotteries involving the former are better than lotteries involving the latter, if they're otherwise exactly the same. Alleged counterexamples to this axiom can be presented in a manner that makes apparent how the relevant judgments contradict this thought. And this often reduces the intuitive plausibility of these judgments significantly. ${ }^{64}$ Second, there's a general strategy to render axiologies that prima facie violate Independence consistent with this axiom. Standard prima facie violations of Independence arise when the value of some state of affairs depends on modal facts-such as facts about what could have happened, or about the probability with which something happened. If this is so, we can take these modal facts to be part of our outcomes. And once we do so, Independence is standardly no longer violated. In other words, we can individuate outcomes in a more fine-grained manner, and thus make axiologies that prima facie violate Independence consistent with this axiom. ${ }^{65}$ This isn't the place to elaborate on this strategy. I'll discuss it in some depth in Section 6.2. There, I mention an important problem for the strategy when we apply it to standard deontic (especially deontological) moral theories. But I argue that this isn't a problem when we apply it to axiologies. As far as I see, the strategy is indeed convincing for standard axiologies. ${ }^{66}$ This isn't to say it works for all possible axiologies. As I'll point out, there are possible axiologies that violate Independence, but not because on these axiologies, the value of outcomes depends on any relevant fine-grained features of states of affairs. And our strategy won't work for these theories. But my sense is that these axiologies are comparatively rare and implausible. So in sum, I think there are axiologies that strictly violate Independence, and to which we should assign some nonzero credence. Hence the axiom is a nontrivial restriction of our framework, or of the plausibility of (2.10) as a general theory of axiological uncertainty. However, since these axiologies are rare and implausible, Independence doesn't seem to diminish the relevance of our argument all too much. Even though it rules out axiologies that strictly violate Independence, the argument still applies to an interestingly large class of theories.

64 As indicated in footnote 51, this has been done e. g. by Savage (1954, $101 \mathrm{ff}$.) and Raiffa (1968, $80 \mathrm{ff}$.) with respect to the example by Allais (1953).

65 For famous applications and defences of this strategy, with respect to the example by Allais (1953), see e. g. Weirich (1986) and Broome (1991, ch. 5).

66 For recent contributions to the debate about the Independence axiom in decision theory and first-order value, see e. g. McClennen (2009), Temkin (2012, 237 ff.) or Buchak (2013, 157 ff.). 
In a similar manner, there are axiologies that strictly violate Continuity. If a theory assigns infinite value to some outcome and finite values to others it will violate Continuity. If we don't put any restrictions on the set of outcomes $X$, then many plausible axiologies are of this form. Take the welfare-theory. On this view, a world that contains beings whose lives are worth living, and only such beings, for an infinite stretch of time, will be infinitely valuable. But there are other ways in which axiologies violate Continuity. An axiology may say that one kind of value lexically dominates another. Consider an axiology on which the value of wellbeing lexically dominates that of beauty-in that a prospect is better than another whenever it expectably leads to more wellbeing, but ceteris paribus better if it expectably leads to more beauty. This welfare/beauty-theory doesn't satisfy Continuity. ${ }^{67}$ More straightforwardly, an axiology may imply a discontinuous behaviour of value with respect to probabilities. Consider an axiology on which, for some $\left.p_{0} \in\right] 0,1\left[\right.$, outcomes that arise with a probability of less than $p_{0}$ are irrelevant for the evaluation of prospects. This axiology will not be continuous. Again, plausibly, we should have some nonzero credence in such axiologies. So the assumption that all axiologies are continuous is a nontrivial restriction. And again, it's an essential aspect of the argument in this book.

But here too, my sense is that at least for finite worlds, axiologies that strictly violate Continuity are comparatively implausible. That's for the same two reasons as with Independence. First, at least for finite worlds, Continuity expresses a very compelling thought. Nothing is infinitely better than anything else; and betterness strictly is a graded and continuous matter. This in itself casts doubt on axiologies that violate this axiom. Second, the strategy of reindividuating outcomes again helps to render standard axiologies that prima facie violate Continuity consistent with it. So again, I think this is a restriction that doesn't diminish the relevance of our argument all too much.

The most problematic axiom is Completeness. And Completeness is problematic indeed. To assume that the value facts are complete is to assume that all prospects are fully commensurable-i.e., that their values are representable by a single value function. But on many axiologies, there's some intratheoretic incommensurability. Some prospects aren't fully commensurable, but compare only roughly. On these axiologies, there are prospects $a$ and $b$, where $a$ isn't determinately at least as good as $b$, and $b$ isn't determinately at least as good as $a$. Indeed, many such axiologies are plausible, and have been prominently defended

67 See e. g. Vallentyne (1993) for a view with this structure. Temkin (2012, $245 \mathrm{ff}$.) also mentions that some outcomes may be 'good enough', or very significantly better than others, so that Continuity fails. I'm not sure whether he'd regard those as instances of the kind of 'lexical betterness' I mentioned. 
by philosophers. ${ }^{68}$ To take a classic example, ${ }^{69}$ suppose that welfare has value, and consider lives of successful musicians and of successful lawyers. It's plausible that for some such pair of lives, it's neither true that the life of the musician is determinately better than that of the lawyer, nor that it's determinately worse, nor that they're precisely equally good. The standard argument for this is the 'small improvement argument'. ${ }^{70}$ Consider some such pair of lives, where it's plausible that neither is determinately better than the other. Now add a small improvement to the life of the musician-an additional successful serenade in place of a bored evening with migraine, say. This new life of the musician is determinately (slightly) better than the former life of the musician. But plausibly, it still needn't be determinately better than the life of the lawyer. So our original lives aren't exactly equally good either. No 'at least as good as'-relation determinately holds between them.

I don't think that Completeness somehow expresses a thought that's independently compelling. And with this axiom, the strategy of reindividuating outcomes won't help. So Completeness at the level of axiologies is a major restriction of the argument from Chapter 2. Fortunately, however, it's a restriction we can overcome within the axiomatic approach, and the broader idea of Expected Value Maximisation. Completeness isn't essential for our main argument. There are representation theorems without the Completeness axiom. These theorems allow our argument to cover axiologies featuring incommensurability. So the plausibility of such axiologies is one major reason to explore such theorems. I'll turn to this in Chapter 6.

\section{The von Neumann-Morgenstern axioms as conditions on m-value}

Consider the assumption that the m-value facts are vNM-conformable. Is this plausible, given the presupposition that all axiologies are vNM-conformable? In many respects, the von Neumann-Morgenstern axioms seem just as plausible with respect to m-value as with respect to first-order value. Some people may question whether $\succeq_{m}$ is even transitive. But since I take m-betterness to be a form of betterness, I again think the transitivity of $\succeq_{m}$ is analytic. In principle, m-value may also violate Independence, even if all axiologies are vNM-conformable. In particular, it's possible that m-value is risk-neutral with respect to purely descriptive uncertainty, but risk-sensitive with respect to axiological uncertainty. But as far as I see,

68 See e. g. Raz (1986, ch. 13) or Broome (1997).

69 See Raz (1986, ch.13).

70 For early instances of the argument, see e. g. Sinnott-Armstrong (1985) or Raz (1986, ch. 13). 
there's no reason why that should be the case-at least none that's somehow specific to the problem of axiological uncertainty. So I think Independence is a plausible axiom about $\mathrm{m}$-value. As with ordinary value, $\mathrm{m}$-value would violate Continuity if it displayed a discontinuous behaviour with respect to probabilities-if there were probability thresholds $\left.p_{0} \in\right] 0,1[$ below which an axiology became irrelevant for determining $\mathrm{m}$-value, say. This could be true even if all axiologies are vNM-conformable. But such thresholds seem implausible, in the context of axiological as in that of purely descriptive uncertainty. So in this regard, Continuity seems plausible too.

However, there's at least one specific reason for why the m-value relation may not be vNM-conformable, even if all axiologies are. That's facts about intertheoretic comparisons. The most problematic assumption in this regard is again Completeness. To assume that $\succeq_{m}$ is complete is to assume that all axiologies are fully commensurable-i.e., that they're all jointly representable by single value functions. But as with intratheoretic comparisons, and even if all axiologies themselves are complete, it might be that there's some intertheoretic incommensurability. It might be that some axiologies aren't fully commensurable, but compare only roughly. If so, there are prospects $\boldsymbol{a}$ and $\boldsymbol{b}$, where $\boldsymbol{a}$ isn't determinately at least as $\mathrm{m}$-good as $\boldsymbol{b}$, and $\boldsymbol{b}$ isn't determinately at least as $\mathrm{m}$-good as $\boldsymbol{a}$. And indeed, this again seems plausible. Suppose that $T_{i}$ is a beauty-theory, and $T_{j}$ a welfare-theory. Even if both $T_{i}$ and $T_{j}$ are complete with respect to $\mathcal{O}$, intuitively, it seems plausible that the value of beauty, according to $T_{i}$, isn't fully commensurable to the value of wellbeing, according to $T_{j}$. For some increase in beauty and some increase in welfare, the value of the former, according to $T_{i}$, is neither determinately greater than that of the latter, according to $T_{j}$, nor determinately smaller, nor precisely equally great. A small improvement argument again seems pertinent. It's implausible that any slight additional beauty, say, would have to tip the balance. No positive 'at least as m-good as'-relation may hold between prospects leading to value-beautytradeoffs, in light of uncertainty about these theories. So the plausibility of such intertheoretic incommensurability is another major reason to explore theorems without the Completeness axiom. Such theorems allow our argument to cover axiologies that aren't fully intertheoretically commensurable.

However, here the problem runs even deeper than with Completeness about axiologies. At the level of first-order value, some prospects on some axiologies may not be fully commensurable. But there doesn't seem to be anything metaphysically problematic about value comparisons, per se. At least some intratheoretic comparisons plausibly do in fact hold. It's not that all plausible axiologies are radically incomplete-or such that $a$ is at least as good as $b$, according to them, only if it's in no respect worse. That's different at the level of m-value. Intertheoretic comparisons seem metaphysically problematic. One might think that no such compar- 
isons hold-that there just aren't any facts, say, about how good certain outcomes would be if some axiologies were true which actually are false. The universe may contain nontrivial value facts about which purely descriptive prospects are better than which. But it just doesn't contain any nontrivial $m$-value facts, or nontrivial facts about which outcomes on which theories are better than which outcomes on which other theories, say. So one might endorse radical scepticism about intertheoretic comparisons, or hold that the $\mathrm{m}$-value relation is radically incomplete-that $\boldsymbol{a}$ is at least as m-good as $\boldsymbol{b}$ only when it's at least as good on all axiologies with nonzero probability. If this is true, the prospect-explication might tell us what it would be for an intertheoretic comparison to be the case. But as a matter of fact, no such comparison holds. Some people have defended this. If they're right, it's little use to have representation theorems allowing for some incompleteness. The idea of Expected Value Maximisation is more fundamentally flawed. The only positive $\mathrm{m}$-value facts are trivial cases, where all axiologies agree. So we need to address the problem of intertheoretic comparisons: provide a positive reason to think that some such comparisons actually hold, and a story about what could ground them. I'll turn to this in the next chapter. To anticipate: I'll argue that intertheoretic comparisons are possible, or that $\succeq_{m}$ isn't radically incomplete. But it isn't fully complete either, even if all axiologies are. Some plausible axiologies are indeed less than fully commensurable.

The problem of intertheoretic comparisons affects the Continuity axiom as well. One might hold that there are axiologies that compare in a lexical wayaxiologies $T_{i}$ and $T_{j}$ such that any positive value-difference between prospects according to $T_{i}$ is greater than any positive value-difference between prospects according to $T_{j}$. This may be so, one might hold, because although there are grounds for intertheoretic comparisons, they're somehow special, or peculiar, and give rise to lexical intertheoretic comparisons. And if two theories compare in this manner, the m-value facts won't satisfy Continuity with respect to them. So to assess Continuity about m-value, again, we have to address the problem of intertheoretic comparisons. To anticipate: on the view of intertheoretic comparisons for which I'll argue, it's implausible that two axiologies compare in a lexical way. So I think (at least for finite worlds) Continuity is a plausible assumption about m-value.

\subsection{The prospect-explications}

Consider our conceptual assumption, the prospect-explications. Are these viable explications of cardinal value? Start with the most fundamental concern. One might think the prospect-explication of intertheoretic comparisons makes the argument from our representation theorem to EVM circular. One might think the ex- 
plication simply defines quantities of value in a way that renders EVM true: that in assuming that intertheoretic comparisons acquire their cardinal significance in the context of axiological uncertainty, I must already assume that EVM is correct. ${ }^{71}$

But this suspicion is misguided. To see this, it may help to distinguish two assumptions involved in the explication. The first assumption is that if there's a theory-dependent utility function, unique up to positive affine transformation, which represents the $\mathrm{m}$-value relation ordinally and each axiology cardinallyand in particular, if $\geq_{m}$ thus satisfies Completeness-all axiologies are somehow fully commensurable, and can somehow jointly be represented by single value functions. This claim doesn't say anything about which value functions jointly represent our theories, or how they compare. It merely says that axiologies are somehow fully commensurable. The second assumption is that the specific value functions that can figure in a representation are then determined by the m-value facts. In my explication, I've joined those two assumptions together. I could have stated them separately. Doing so makes more vivid how weak a presupposition the prospect-explication of intertheoretic comparisons really is.

Consider the first assumption first. This is simply a claim about what (perhaps among else) it means that two theories are comparable. And though it is necessary for EVM, it certainly doesn't presuppose EVM. Moreover, the assumption doesn't seem very problematic. If there's a theory-dependent utility function, unique up to positive affine transformation, which represents $\mathrm{m}$-value ordinally and each axiology cardinally, there's a unique way in which axiologies weigh against each other to determine complete m-value facts. In any sense that's relevant for the problem of axiological uncertainty, this means all axiologies are 'comparable' in some way. So grant this first assumption. Then, if there is such a function, the respective state-wise utility functions must be positive affine transformations of the value functions of our theories. That is, if $G_{i}$ are our value functions, there must be $s_{i}$ and $t_{i} \in \mathbb{R}, s_{i}>0$, such that for all prospects $\boldsymbol{a}$ and $\boldsymbol{b}$,

$$
\boldsymbol{a} \geq_{m} \boldsymbol{b} \quad \text { iff } \quad \sum_{i \in I, x \in X} \boldsymbol{a}(i, x)\left[s_{i} G_{i}(x)+t_{i}\right] \geq \sum_{i \in I, x \in X} \boldsymbol{b}(i, x)\left[s_{i} G_{i}(x)+t_{i}\right] .
$$

Hence the only alternative way in which our value functions could determine the $\mathrm{m}$-value relation would be if, apart from their probabilities, one theory had systematically and constantly more weight in determining $\mathrm{m}$-value than another (in

\footnotetext{
71 This objection seems to be raised by Andrew Sepielli (2009, 27): 'The main problem with [the prospect-explication of intertheoretic comparisons] is that it simply assumes the rationality of maximizing [expected value] under normative uncertainty. But this is a position that should be argued for independently of one's solution to the [problem of intertheoretic comparisons], not merely assumed as a means to solving the problem.'
} 
that $t_{i} \neq t_{j}$ or $s_{i} \neq s_{j}$ for some $i$ and $j$ ). So the second assumption-that intertheoretic comparisons acquire their specific cardinal significance in the context of axiological uncertainty-doesn't rule out risk-aversion, or the quasi-deontological or virtue-ethical accounts sketched in Section 2.4. It only rules out such constant unequal weighing. And although EVM presupposes that m-value isn't determined by such unequal weighing, to turn this final step into a matter of definition is again by no means to assume EVM. What may be questioned is whether there is a utility function that represents $\succeq_{m}$ in the relevant way-i. e., whether $\succeq_{m}$ satisfies the conditions of our theorem, and particularly Completeness. These conditions are doing the main work guaranteeing intertheoretic comparability. Once they're in place, the explication of intertheoretic comparisons doesn't add much, and certainly not enough to warrant a charge of circularity.

Here's a second objection against our explications. I suggested in Section 1.2 that we don't use cardinal comparisons in ordinary language, that we thus can't find out what they really mean, or that there's no uniquely privileged definition of them. Instead, we should pick a useful explication. One might object to this. One might claim that there is a privileged derivation of cardinal comparisons. In particular, one might say we have a pretheoretic understanding of (ordinal) comparisons of value-differences, or facts like

(M) according to $T_{i}$, the value-difference between $x$ and $y$ is greater than the valuedifference between $z$ and $t$.

Now a comparative ranking of value-differences among a set $X$ of outcomes doesn't always entail a cardinal concept of value. Suppose $X$ has only three members, and that according to $T_{i}$, the difference between the best and the second best outcome is greater than the difference between the second best and the third. Clearly, this isn't enough to determine cardinally how much greater the first difference is than the second.$^{72}$ However, under some conditions, difference comparability is enough to imply cardinal measurability: if $X$ is rich enough, facts like (M) determine a utility function unique up to positive affine transformation representing $T_{i}$. For instance, on one condition introduced by Kaushik Basu $(1983,197)$, intuitively, if you're able to compare value-differences in a set as rich as the rational numbers, then such ordinal comparability of value-differences implies cardinal measurability. ${ }^{73}$ So then an understanding of value-difference

72 See e. g. Bossert $(1991,212)$ for such an example.

73 More precisely, Basu $(1983,197)$ proved the following. Let $X$ be a set of outcomes, $u$ a realvalued utility function on $X$, and $\Omega$ a set of real-valued functions on $u(X)$. Think of functions in $\Omega$ as permissible transformations of $u$-transformations, say, that also represent the relevant 
comparisons is enough to provide a cardinal concept of value. And since facts like (M) must be accepted by everyone as primitives (the objection goes) this is the privileged explication of cardinal value. ${ }^{74}$

I'm not convinced by this proposal. For one thing, it seems dubious that we understand statements like (M). They might be unproblematic as long as valuedifferences count the same no matter in what time and place, and in what prospect they appear. Then these contexts help us understand them. But they become problematic when these contexts vanish. Suppose you say that for you the ex post value of money increases linearly: that the value-difference between $€ n$ and $£(n+100)$ is the same for any $n \in \mathbb{N}$. And suppose you say you are risk-averse about this value: that a certain prospect of $€ n$ is always better than a $50 \%$ chance of getting $£ 2 n$ or nothing. Then you arguably need to tell us in what sense you value money linearly, ex post, instead of assigning it diminishing marginal value. Our grasp of value-difference comparisons isn't robust enough to make this clear. Or in short, we don't just lack an unmediated understanding of cardinal comparisons like (B) (from page 5). We also lack an unmediated understanding of facts like (M).

But we can even grant that we understand intratheoretic value-difference comparisons. This still doesn't get us very far. Whether a prospect has a higher expected value than another also depends on intertheoretic value-difference comparisons, or facts like

(N) the value-difference between $x$ and $y$, according to $T_{i}$, is greater than the value-difference between $z$ and $t$, according to $T_{j}$.

And it seems uncontroversial that we have no direct grasp of such facts. So primitivism about (M) will anyway be insufficient to formulate EVM. In the next chapter, as a possible solution to the problem of intertheoretic comparisons, I'll introduce fitting attitude accounts of value. I'll consider whether an ordering of the strength of attitudes corresponding to value-differences might provide a cardinally significant concept of value. But I'll argue that (at least with respect to intertheoretic

ordering of $X$ that $u$ represents. Say that $(u \mid \Omega)$ has cardinality if for all $f$ in $\Omega$ there are $s$ and $t \in \mathbb{R}$, $s>0$, such that for all $a$ in $u(X), f(a)=s a+t$. In other words, $(u \mid \Omega)$ has cardinality if only positive affine transformations are permissible transformations of $u$. Say that $(u \mid \Omega)$ has difference comparability if for all $f$ in $\Omega$ and for all $a, b, c$ and $d$ in $u(X), a-b \geq c-d \Leftrightarrow f(a)-f(b) \geq f(c)-f(d)$. In other words, $(u \mid \Omega)$ has difference comparability if only transformations that preserve ordinal difference-comparisons are permissible transformations of $u$. Now if $u(X)$ is dense in a connected subset of $\mathbb{R},(u \mid \Omega)$ has cardinality if and only if it has difference comparability. (The connected subsets of $\mathbb{R}$ are just the intervals on $\mathbb{R}$, and $\mathbb{Q}$ is dense on $\mathbb{R}$. Thus the intuitive formulation above approximates this result.) See e. g. Bossert and Stehling (1994) for another condition; e. g. Bossert (1991), Bossert and Weymark (2004, 1126 ff.) or Bossert et al. (2005, 34 f.) for a general discussion. 74 I thank Ralf Bader for mentioning this worry to me. 
comparisons) that's ultimately dubious. So ultimately, I don't think we can find any better-let alone a uniquely privileged and 'correct'-explication of cardinal value in terms of value-differences.

There's a third worry about the prospect-explications. One might think these explications get the order of explanation wrong. Consider intratheoretic comparisons, and take two prospects $a$ and $b$, where (according to the true axiology) $a$ is better than $b$. Intuitively, it's not that outcomes compare in a certain way because, as a brute matter of fact, $a$ is better than $b$. Rather, $a$ is better than $b$ precisely $b e$ cause outcomes compare in some way. So my explication seems to put the cart before the horse. It explains comparisons in terms of betterness facts, whereas actually such facts seem explained by comparisons. And the same worry arises in the intertheoretic case. Intuitively, it's not that axiologies compare in a certain way because certain m-value facts hold. Rather, such facts hold because axiologies compare in that way. ${ }^{75}$

Let me say three things in reply to this objection. For simplicity, I'll only discuss the intratheoretic case. But what I say applies mutatis mutandis to intertheoretic comparisons. First, a point of clarification. The prospect-explication doesn't entail that cardinal comparisons are grounded in value facts, or that it's value facts that explain these comparisons. It assumes that for a cardinal comparison to hold just is for a certain value fact to be the case. It assumes an identity rather than a grounding relation between comparisons and value facts. So to the extent that one finds the alleged grounding relation objectionable, the worry simply misses our explication.

Second, nothing in the prospect-explication implies that the value facts themselves must be fundamental, or not grounded in anything. They may well have grounds. Suppose according to the true axiology, both natural beauty and wellbeing have value, that prospect $a$ leads to the destruction of some natural beauty with a probability of $\mathbf{0 . 4}$, and to some benefits for Antonio with a probability of 0.6 , and that prospect $b$ represents the status quo:

Tab. 3.3: Example to illustrate prospect-explications.

\begin{tabular}{lll}
\hline & $\boldsymbol{a}$ & $\boldsymbol{b}$ \\
\hline 0.4 & destruction of natural beauty & status quo \\
0.6 & benefits for Antonio & status quo \\
\hline
\end{tabular}

75 This objection is made in MacAskill $(2014,146)$; it's often raised against similar explications in slightly different contexts (see e. g. Eriksson and Hájek 2007, 207). 
Now suppose that $a$ and $b$ are equally good. This fact needn't be brute. It may have grounds. What these are will depend partly on the correct metaethics. Perhaps the fact that $a$ and $b$ are equally good is grounded (in the manner of something like a divine command theory) in the fact that God would be indifferent between them. Or perhaps it's grounded (in a constructivist way) in the fact that the best systematisation of our axiological intuitions implies that $a$ is equally as good as $b$. Or perhaps this fact is simply grounded (in a realist manner) in the fact that $a$, while having a good probability of leading to something mind-independently intrinsically valuable, also has a decent probability of destroying something mindindependently intrinsically valuable. And so on. What the explication rules out is only that our comparison is grounded in the cardinal fact that the value of benefiting Antonio is 2/3 of the disvalue of destroying that natural beauty. And that's, again, because it assumes an identity relation between value relations and cardinal comparisons. Something similar holds for intertheoretic comparisons. The prospect-explications don't presuppose that the m-value facts are brute. It's compatible with their having grounds, as long as these grounds aren't themselves cardinal comparisons. To show that there are such grounds, or what they can be, we'll have to address the problem of intertheoretic comparisons. But it's important to note that the explication itself is neutral on this.

Thirdly and most importantly, the objector's claim that value facts are explained by cardinal value comparisons presupposes an independent cardinal notion of value. So if her objection is to get off the ground, she needs an alternative understanding of cardinal value comparisons. And if my reply to the previous objection is sound, this understanding is only to be had via some alternative explication. There are alternative explications. The objector could assume, say, that value acquires its cardinal significance in the context of weighing goods over time. But presumably, all these explications will stipulate an identity where the objector wants to see a grounding relation. On the time-explication, say, the fact that two value-differences coming at different times count the same in determining the goodness of the history of the world isn't explained by the fact that these differences are the same. Rather, for the former fact to hold just is for the latter to do so. And to the objector's ear this will presumably sound as objectionable as our equivalent claim about prospects. So by her own lights, nothing is gained with the move from one explication to the other. But this tu quoque-response doesn't imply that the entire project of getting a cardinal concept of value is doomed. It reveals that the alleged order of explanation that the objector stipulates is deceptive. It's just not true that there are primitive cardinal comparison facts that ground value facts concerning prospects or times. 
If all of this is right, there doesn't seem to be anything wrong with the general approach embodied in the prospect-explications. ${ }^{76}$ But there's a final worry, internal to this approach. It doesn't challenge the very idea of explicating value comparisons in terms of m-value facts. But it suggests the specific explications I've given still leave important questions unanswered. For one thing, even if they're viable as far as they go, they only explicate what precise comparisons mean. But if it's right that many plausible axiologies allow for intratheoretic incommensurability, we also need an explication of what rough intratheoretic comparisons mean. And if it's right that many axiologies give rise to intertheoretic incommensurability, we need an explication of what rough intertheoretic comparisons mean. For another thing, these explications don't yet tell us the meaning of axiological probabilities. As indicated, these probabilities require explication as well. And the problem of explicating probabilities isn't separate from that of explicating comparisons. If we don't take probabilities as primitives (as I've done), we need to separate probabilities and values-or cases where an axiology has a low probability but an inflated value function from cases where it has a high probability but a deflated value function. If we can't get this separation, we ultimately can't get a viable explication of either probabilities or interthereotic comparisons. So we have to refine these explications further.

I think these are important worries. They show that the Basic Representation Theorem is still too simple. It must be extended to allow for an explication of probabilities, and for one of incommensurabilities. I'll turn to this in Chapters 5 and 6 respectively.

\subsection{Conclusion: decision theory and $\boldsymbol{m}^{2}$-value}

In sum, there are three issues we need to address to validate the argument from the Basic Representation Theorem. First and most fundamentally, we need to show that intertheoretic comparisons aren't metaphysically dubious-that there can be facts that ground such comparisons, or that such comparisons can hold.

76 Ittay Nissan-Rozen (2015) raised a specific objection related to the axiomatic approach. He suggests that if we adopt the decision-theoretic explication of intratheoretic comparisons for our orderings $\geq_{i}$, we're forced to conclude that intertheoretic comparisons are impossible-because the utility functions representing our theories will be unique only up to positive affine transformation, and so intertheoretic comparisons aren't fixed. But that's simply false. Nissan-Rozen is right insofar as an ordering $\succeq_{i}$ in itself can't provide the information for intertheoretic comparisons. But this doesn't rule out the possibility that other facts could provide it-e. g., facts about how this theory weighs against other theories under axiological uncertainty. 
This is to confirm that the m-value relation isn't radically incomplete, and the idea of EVM not fundamentally flawed. And it's to underpin that the prospectexplications don't presuppose a dubious order of explanation, or fundamentality of the m-value facts. Second, we need to provide a representation theorem that doesn't take axiological probabilities as primitives. This is to show that we can explicate the equally problematic notion of quantitative probabilities, and separate probabilities and values effectively. Third, we need to devise a representation theorem without the Completeness axiom, either at the level of $\geq_{i}$ or $\geq_{m}$. That's to allow for axiologies that feature some intratheoretic incommensurability, and for axiologies that yield some intertheoretic incommensurability. And it's to extend our definitions to explicate rough comparisons too. In the remainder of the book-Chapters 4, 5 and 6 respectively-I'll address these three issues in turn.

To end this chapter, let me note two negative upshots of our discussion for the project of extending EVM beyond a theory of uncertainty about vNM-conformable axiologies. Both of them follow from the fact that if one of our axiologies isn't vNM-conformable, our m-value facts arguably aren't vNM-conformable eitheror more generally, from the fact that if one of our normative assumptions isn't vNM-conformable, the meta-facts about what you ought to do under uncertainty about it arguably aren't so either. First, this has an implication for how plausible the axioms are as axioms of decision theory, or the theory of rational preferences. If m-value isn't generally vNM-conformable, but is so only when restricted to vNM-conformable axiologies, then your preferences arguably needn't be generally vNM-conformable either. You can care about m-value in your preferences. So the von Neumann-Morgenstern axioms aren't fully general constraints on your preferences. Indeed, I suggested that it's reasonable to have nonzero credences in some non-vNM-conformable axiologies. So it's reasonable to violate the von Neumann-Morgenstern axioms in your preferences. We can generalise this gloomy upshot beyond axiological uncertainty. Any uncertainty about the axioms at the level of first-order value or preferences implies that they're false as general, or higher-order constraints on decision-making in the face of uncertainty. Their mere doubtfulness implies their strict falsity. This may be the most straightforward way to criticise the axioms of decision theory. It's enough to show we can't be certain about them. And this seems almost indisputable. ${ }^{77}$

Second, the abovementioned fact has an implication for how general the axiomatic argument for EVM can be. As mentioned in Chapter 1, we might not be

77 For an interesting general exploration of 'uncertain preferences' (though not of the specific problem I mention), see e. g. Schulz (2020). 
certain about the true theory of axiological uncertainty. So we might need a theory of uncertainty about theories of axiological uncertainty-a theory of $\mathrm{m}^{2}$-value, and so on. Now one might think if EVM is a plausible theory of m-value, it's also a plausible theory of $\mathrm{m}^{2}$-value. And one might think the axiomatic argument I've provided for EVM as a theory of m-value can straightforwardly be extended to EVM as a theory of $\mathrm{m}^{2}$-value-using theories of axiological uncertainty instead of axiologies, and the $\mathrm{m}^{2}$-value relation instead of the $\mathrm{m}$-value relation. Unfortunately, however, this is true only to a very restricted extent. The Basic Representation Theorem presupposes that all first-order theories are vNM-conformable. And it shows that in this case EVM is the only meta-theory that's vNM-conformable and satisfies the Pareto Condition. Accordingly, on the level of $m^{2}$-value, a similar argument will presuppose that all theories of $\mathrm{m}$-value are vNM-conformable. But as the theorem itself shows, apart from theories that violate the Pareto Condition, EVM is the only theory of this kind. And we saw that the Pareto Condition is very plausible. So the set of theories of $\mathrm{m}$-value that are vNM-conformable but violate the Pareto Condition is not a very interesting set. The more interesting set of theories are those that aren't vNM-conformable. And to those the argument doesn't apply. It follows that my argument, applied to $\mathrm{m}^{2}$-value instead of m-value, is extremely severely limited. More generally, we can't simply assume that the same kind of argument will in principle be available, and of the same importance, on each level of value. If representation theorems are as important as I'm arguing in this booknot only in defending, but in even defining our views-then higher-order problems of uncertainty may be even more serious than it might have seemed. We may not even be able, in any interesting sense, to define our views of uncertainty about theories of axiological uncertainty. 